\title{
Modelo Integra/Erantsi de acompañamiento psicosocial para personas en procesos de integración social
}

\author{
Carmen Vicioso Etxebarria \\ Asociación T4 \\ carmen@asociaciont4.org
}

\section{Daimel Castillo-González}

Asociación T4

\begin{abstract}
Bazterkeria-egoeran dauden eta askotariko osasunarazoak dituzten lagunen zailtasunak are eta larriago eta kronikoagoak izaten dira maila indibidualean (osasun mentala), familia- (gatazkak, hausturak) eta gizarte-mailan (bazterkeria, isolamendua). Hori dela-eta, berariazko laguntzatresnak ezartzea premiazkoa da, gizarteratzeprozesuetan mantendu eta bizi-kalitatean eta gizarteratzean aurrera egiteko. Artikulu honetan, Integra/Erantsi eredua aurkezten da, adikzioekin edo gaixotasun psikiatrikoekin lotutako askotariko gizarte- eta osasun-arazo dituzten pertsonei gizarteratze-prozesuetan laguntza psikosoziala bideratzen dena. Azken urteotan, eredua laguntzagida batean islatu da, eta Hiesaren aurkako $\mathrm{T}_{4}$ Elkarteak ezarri. Erakundearen erabiltzaileen artean, babes, harreman eta doikuntza sozialaren eta antsietatearen inguruan hobekuntza nabarmenak igarri dira.
\end{abstract}

\section{GAKO-HITZAK:}

Lagun egitea, gizarteratzea, bizi-kalitatea, behar konplexuak.
Las personas en exclusión social que padecen múltiples problemas de salud suelen experimentar un agravamiento y cronificación progresivas de sus dificultades a escala individual (salud mental), familiar (conflictos, rupturas) y social (exclusión, aislamiento), lo cual hace necesario establecer herramientas de acompañamiento específicas que les permitan mantenerse en los procesos de inserción y avanzar en su calidad de vida e integración social. Este artículo presenta el modelo Integra/Erantsi, destinado a facilitar el acompañamiento psicosocial en procesos de inclusión a personas con múltiples problemas sociales y de salud asociados a adicciones o a enfermedades psiquiátricas. El modelo se ha plasmado en una guía de acompañamiento y se ha implantado en los últimos años en la Asociación T4 de Lucha contra el Sida, habiéndose observado mejoras significativas en las dimensiones de apoyo social, relaciones sociales, ajuste social y ansiedad entre las personas usuarias de la entidad.

\section{Palabras ClaVe:}

Acompañamiento, integración social, calidad de vida, necesidades complejas. 


\section{Introducción}

En este artículo se realiza una aproximación teórica al acompañamiento psicosocial a la integración social. La primera parte recoge los fundamentos de la Ley $12 / 2008$, de 5 de diciembre, de Servicios Sociales en el ámbito de la inclusión social, así como el procedimiento de valoración de la exclusión social y la intervención social en los servicios y prestaciones sociales para la inclusión social en Euskadi. En la segunda parte se analizan los retos que el sistema establecido plantea para los y las profesionales y personas usuarias. Por último, la tercera parte del artículo está destinada a describir y desarrollar el modelo de acompañamiento psicosocial a la integración social Integra/ Erantsi. Se describen los objetivos y principios básicos del modelo, haciendo especial énfasis en la relación de ayuda, la metodología de acompañamiento psicosocial, las estrategias e intervención y la evaluación de los resultados.

\section{El acompañamiento para la inclusión social en Euskadi: la Ley 2008 de Servicios Sociales}

\subsection{Fundamentación de los servicios sociales para la inclusión en la ley de servicios sociales}

Los servicios sociales para la inclusión en Euskadi se rigen por el marco conceptual que centra la atención en las necesidades de las personas (Gobierno Vasco, 2008), con los siguientes supuestos:

- Calidad de vida y atención centrada en la persona (ACP). Este modelo se constituye a partir de la individualización y adaptación a las necesidades y expectativas de las personas usuarias de los servicios sociales.

- Promoción de la autodeterminación. Implica reconocer a las personas usuarias de los servicios sociales como individuos con fortalezas, capacidades y preferencias, y poner estas en el centro de los cuidados y servicios que reciben.

- Reducción de daños y baja exigencia. Conceptualización de la adicción como un problema psicosocial, y modelo teórico basado en los derechos humanos, que es paradigma de la integración de teoría y práctica, de atención individual y comunitaria, de complementariedad del modelo médico y social.

- Activación inclusiva y ciudadanía activa. Un modelo de este tipo requiere reconocer la multidimensionalidad de la inclusión y sus implicaciones. Del mismo modo, la apuesta por una concepción multidimensional de la inclusión implica hablar de interseccionalidad, es decir, de cómo las diversas discriminaciones y opresiones que padece una persona interactúan de manera simultánea, configurando su identidad y su realidad.
- Perspectiva de género. Implica una consideración más estructural de la perspectiva de género en los servicios y centros para la inclusión social, promoviendo un enfoque basado en las necesidades, expectativas, capacidades y preferencias de cada persona atendida desde el punto de vista de la desigualdad de género.

- Atención en la comunidad. Se ha aceptado de forma casi unánime el gradual abandono de las grandes instituciones residenciales y su sustitución por servicios de base domiciliaria o por soluciones residenciales dispersas y de pequeño tamaño, a fin de permitir que las personas en situación de vulnerabilidad puedan vivir, con garantías, formando parte de la comunidad.

El proceso básico en torno al que se articula la intervención social es el proceso relacional (Fantova, 2007). No solo porque es el canal a través del cual se accede a una variedad de recursos, prestaciones o programas, sino porque en numerosas ocasiones, el servicio es la misma relación. Una relación cualificada que trata de acompañar a las personas en procesos vitales; una relación que apoya y facilita la vida, a través de dar soporte a la clarificación personal, a la toma de decisiones, que ayuda en la formulación de proyectos a corto y a largo plazo y que apoya su realización, su valoración y su redefinición.

Las personas se encuentran en situación de exclusión social cuando sus condiciones de vida y convivencia se están viendo afectadas por múltiples carencias que persisten en el tiempo y que, al acumularse, provocan la existencia de una situación de exclusión social que está relacionada directamente con los recursos personales, los recursos relacionales y los recursos materiales (Gobierno Vasco, 2013).

Las personas en esta situación tienen serias dificultades para comunicarse y para participar en la vida social y comunitaria. Tienen problemas en sus competencias y habilidades sociales y personales para poder asumir su propia responsabilidad en la cobertura de sus necesidades. Todo ello las lleva a un aislamiento comunicacional y relacional.

La exclusión social no se refiere solo a la insuficiencia de recursos financieros, ni se limita a la mera participación en el mundo del empleo; se hace patente y se manifiesta también en los ámbitos de la vivienda, la convivencia, la educación, la salud o el acceso a los servicios (Fantova, 2017).

\subsection{La valoración de la exclusión social de servicios sociales para la inclusión en la ley de servicios sociales}

El primer paso que se ha de producir para acceder al derecho a los servicios sociales para la inclusión es la valoración (Gobierno Vasco, 2017). La valoración comprueba la existencia de una situación de necesidad: una situación de riesgo de exclusión, 
una situación reconocida de exclusión, o una situación de urgencia social que pueda atenderse desde los servicios sociales (Gobierno Vasco, 2013). Además, se tiene en cuenta para la valoración la intensidad del apoyo formal o atención requerida, así como la disponibilidad de apoyos prestados por la red sociofamiliar. En resumen, las necesidades de inclusión se establecen en base a la valoración integrada de la autonomía funcional y la integración relacional (Gobierno Vasco, 2013).

El proceso de valoración permite a las personas contrastar su situación, explicitar sus objetivos de autonomía e integración social y contar con una figura profesional de referencia que, a lo largo del proceso, sea capaz de ofrecerle apoyo. Estos objetivos se concretan en un plan individual de intervención que incluye prestaciones de acompañamiento social, psicoeducativas, socioeducativas, y psicosociales. Estos criterios se recogen en el instrumento de valoración de la exclusión social (Gobierno Vasco. 2017):

- Criterio 1

- Dimensión relacional.

- Dimensión social.

- Dimensión personal.

- Dimensión residencial, espacial.

- Criterio 2

- Ámbito económico-laboral

- Convivencial

- Personal

- Salud

Si se cumple el criterio uno, se debe comprobar que al menos dos de los cuatro ámbitos superan el porcentaje mínimo de carencia para determinar la existencia de exclusión social. Es decir, para que se valore una situación con diagnóstico de exclusión social, se deben cumplir los criterios uno y dos. En el caso de que se considere que la persona se encuentra en situación de exclusión social, también podremos calificar la intensidad de dicha situación. La intensidad de la exclusión social puede ser:

- Leve: desarraigo inicial o leve. Personas que disponen de contactos frágiles con sus familiares o red de apoyo.

- Moderada: fase avanzada de desarraigo. Personas que han roto sus lazos sociales básicos.

- Grave: no participa en la sociedad. Precariedad o ausencia de relaciones sociales y afectivas.

\subsection{La intervención social en los servicios sociales para la inclusión de la Ley 2008 de Servicios Sociales}

La Ley 12/2008 de Servicios Sociales denomina intervención social a la actividad que se realiza en los servicios sociales, señalando que se orienta a la promoción y protección de la autonomía personal y la integración comunitaria en todas las personas, familias y grupos, desarrollando una función promotora, preventiva, protectora y asistencial, a través de prestaciones y servicios de naturaleza fundamentalmente personal y relacional (Gobierno Vasco, 2008).

La intervención social consiste en la actividad profesional que tiene como finalidad prevenir, corregir o paliar desajustes en lo relativo a la interacción de las personas, con sus dos caras o dimensiones: autonomía funcional e integración relacional.

- Autonomía funcional para la vida diaria, que comprendería desde el autocuidado o cuidado en la ejecución de las actividades básicas de la vida diaria hasta la utilización de las funciones cognitivas y emocionales para la toma de decisiones sobre la propia vida y su futuro (autodeterminación, autoorganización o autogobierno), pasando por habilidades sociales para las relaciones cotidianas.

- Integración relacional, que se referiría tanto a los vínculos familiares como a otras redes comunitarias presenciales o virtuales, actuales o posibles (todas ellas, relaciones primarias), fuentes primordiales de apoyo social (LópezArostegi, 1970).

La intervención social, por tanto, estaría llamada a obtener resultados, entendidos como cambios factibles, evaluables y evidentes en la interacción de las personas, en ese ajuste dinámico entre la autonomía funcional para la vida cotidiana y la integración relacional en redes primarias (Fantova, 2017).

La intervención ajustará su nivel de exigencia e intensidad a las necesidades dinámicas y periódicamente valoradas de las personas usuarias. A continuación se especifica cada criterio (Diputación Foral de Bizkaia, 2019):

- Intensidad: la intensidad de los servicios vinculados a procesos de inclusión social se determinará por su contenido prestacional, debiendo entenderse la intensidad como la dedicación y el número de horas de prestaciones técnicas directas que precisa la persona usuaria, determinadas mediante el diagnóstico social especializado.

- Exigencia: la exigencia hace referencia, con base en los indicadores del diagnóstico social especializado, a la posición de la persona respecto de su situación y al grado de compromiso que es capaz de asumir en relación con ella y con su proceso de inclusión.

El catálogo de servicios sociales para la inclusión de la ley de servicios sociales prevé dos niveles de intervención: primario o comunitario, y secundario o especializado (Gobierno Vasco, 2008). El nivel primario ofrece, entre otros, servicios de baja intensidad de apoyo. El nivel secundario o especializado ofrece servicios de mayor intensidad 
de apoyo. Este mapa de recursos responde a un marco lógico basado en los procesos de inserción, y se despliega dentro de un plan individual de atención cuya meta es la inclusión activa de las personas en la sociedad. Las personas en situación de exclusión social grave acceden a los servicios de atención secundaria, de mayor intensidad de atención.

El catálogo de servicios de la Ley 12/2008 asigna, en general, los servicios considerados como de atención primaria a los ayuntamientos y los considerados de atención secundaria a las diputaciones forales (Gobierno Vasco, 2008).

Los servicios de intervención social se diferencian también en función de otros criterios, como pueden ser los objetivos de la intervención, el nivel de exigencia o la población potencialmente atendida (Kortabarria et al., 2018).

El catálogo también prevé el espacio y la coordinación para atender las necesidades sociosanitarias de la población en situación de exclusión social y deterioro de salud. Dada la naturaleza cada vez más compleja y plural de las diversidades individuales y colectivas que confluyen en el ámbito de los servicios sociales, se constata la necesidad de reforzar la evaluación y la promoción de buenas prácticas adaptadas a esta complejidad, integrales, basadas en la evidencia científica, y con capacidad de influencia social (Fantova, 2017).

\subsubsection{Los servicios sociales en la Ley $12 / 2008$ de Servicios Sociales para personas con situación de exclusión social grave}

Los servicios sociales destinados a personas en situación de exclusión social grave atendiendo a su cobertura son fundamentalmente de tres tipos: diurnos, nocturnos o residenciales (Gobierno Vasco, 2008).

\section{Sus objetivos son:}

1. Mantener la autonomía personal de las personas usuarias y, en lo posible, fomentar el desarrollo de esta y evitar su deterioro.

2. Potenciar su desarrollo personal, sus capacidades, habilidades y experiencias.

3. Ofrecer a las personas usuarias oportunidades de integrarse, participar en el entorno comunitario y utilizar los servicios comunitarios tomando parte en actividades culturales, sociales y recreativas de su entorno.

4. Promover el mantenimiento y refuerzo de sus redes familiares y sociales de apoyo.

En los centros de día y los centros nocturnos, los requisitos que se deben cumplir son los siguientes:

- Requerir un apoyo de media o alta intensidad para realizar un itinerario de inclusión social.
- No padecer trastornos de conducta, ni presentar comportamientos que puedan perturbar gravemente el funcionamiento del servicio o la normal convivencia en él, o que supongan riesgo para la propia persona, otras personas usuarias 0 las y los profesionales.

En los centros residenciales, los requisitos específicos que se deben cumplir son los siguientes:

- Contar con un mínimo de habilidades sociales y relacionales para la convivencia.

- Requerir una intensidad de apoyo media o alta, en función de la modalidad del servicio al que se solicite el acceso.

\subsubsection{Prestaciones técnicas para el acompañamiento a personas en situación de exclusión social grave}

En la actualidad, el paradigma que se va imponiendo internacionalmente en la intervención social es el de la atención integrada, coincidente con el propuesto por la ley vasca, en el sentido de que no trata de deconstruir los ámbitos sectoriales ni de crear "espacios" intermedios entre sectores: las prestaciones se caracterizan por cuidar la continuidad de la intervención y los itinerarios intersectoriales, así como la integración intersectorial (Fantova, 2017).

\section{Acompañamiento social}

El acompañamiento es la premisa básica sobre la que se asienta la ley de servicios sociales en su definición de la naturaleza u objeto de la labor de los servicios sociales (Casado, 2010). Es la prestación más genuina y característica de los servicios sociales. La persona participa, en interacción con un profesional cualificado, en una relación de ayuda que contribuye a mejorar el desenvolvimiento autónomo y la integración social (Gobierno Vasco, 2008). Permite a las personas contrastar su situación, explicitar sus objetivos de autonomía e integración social, y contar con una figura profesional de referencia que a lo largo del proceso, sea capaz de ofrecerle apoyo. Será de mayor a menor intensidad en función de las necesidades y se podrá ir variando, deseablemente reduciendo la intensidad del acompañamiento requerido.

\section{Intervención socioeducativa}

Puede desarrollarse a nivel individual, grupal, familiar, o comunitario, y en diversos contextos: atención en el domicilio familiar, educación doméstica, y orientación a personas o familias en crisis. La intervención socioeducativa tiene una acción preventiva, en la detección precoz, e identificación de necesidades y carencias en los contextos donde se producen (Burgos et al., 2019). Aunque la intervención socioeducativa se pueda entender como "propia o característica" del educador o educadora, esto no debiera significar que la intervención socioeducativa sea exclusiva de una figura profesional. 


\section{Intervención psicosocial}

El acompañamiento psicosocial puede ser de varios tipos (Gobierno Vasco, 2018; SIIS Centro de Documentación y Estudios, 2013):

- Apoyo instrumental: información, orientación y mediación para el acceso a servicios de otros sistemas así como a redes sociofamiliares de apoyo.

- Emocional: contención, soporte emocional, y orientación en situaciones de crisis.

- Relacional: escucha activa, adquisición de habilidades para mantener o establecer relaciones sociales, $y / 0$ personales, generando oportunidades para el establecimiento de relaciones.

Específicamente, sus objetivos son los siguientes:

- Promover las condiciones personales, familiares, sociales, y comunitarias, y del entorno, que potencien un estilo de vida adaptativo y autónomo.

- Promover los cambios en aquellos factores personales, familiares, sociales, comunitarios, y del entorno dirigidos a reducir o eliminar los factores asociados a situaciones de vulnerabilidad o dificultades.

- Prevenir y paliar las consecuencias psicológicas derivadas de la situación de vulnerabilidad o dificultad social.

- Abordar los factores de tipo psicológico que dificultan a las personas, familias o grupos su integración social, y por ende un estilo de vida adaptativo y autónomo.

- Optimizar el apoyo profesional en su función de ayuda, haciéndolo accesible y competente, así como los apoyos de las redes del sistema informal y natural para maximizar su efecto amortiguador del estrés.

Incluye intervenciones de escucha activa y contención emocional o, también, intervenciones en funciones psico-afectivas, incluyendo en su caso ayudas para el manejo de trastornos de comportamiento.

En línea con lo referido en la ley de servicios sociales con respecto a los criterios de la intervención social dirigida a personas en situación de exclusión social grave, las estrategias de intervención serán de alta intensidad y baja exigencia. El fin último es lograr la autonomía funcional máxima y la integración relacional óptima. A medida que se aumenta la autonomía funcional y la integración relacional mejora, se modula el nivel de exigencia e intensidad de las intervenciones.

\section{Retos en la intervención social en el ámbito de la exclusión social grave}

Según el enfoque de la atención integrada (Fantova, 2018), el reto principal es la falta de perspectiva ecológica, con un enfoque poblacional o una aproximación estructural desde el momento en que hemos entendido que es fundamental intervenir con los individuos pero que también lo es incidir en sus entornos familiares, comunitarios y sociales en general y multidimensional.

Las personas en situación de exclusión social grave se encuentran generalmente en procesos de larga duración, y pueden tener una evolución hacia la inclusión social o pueden evolucionar a procesos de mayor dependencia o discapacidad en caso de que exista un deterioro físico, cognitivo o funcional irreversible.

La concepción multicausal de la exclusión exige desarrollar respuestas institucionales que reconozcan esta multidimensionalidad (Seco Revilla, 2018). Esto implica articular un modelo de derechos sociales basado en el desarrollo comunitario de todas las dimensiones sociales vitales, superando los recursos orientados a la capacitación o la cualificación, e impulsar la orientación hacia el empoderamiento y la defensa de derechos, vinculados intrínsecamente con la dignidad de la persona.

Sin embargo, en la práctica, los servicios sociales para la inclusión no han logrado alcanzar el desarrollo o la madurez como sistema de los servicios destinados a las personas con discapacidad, con dependencia y/o en situación de desprotección (SIIS Centro de Documentación y Estudios, 2013). Entre las razones que explican ese menor desarrollo se hace hincapié en las características de la población destinataria, tanto por la invisibilidad social de la población atendida (inmigrantes, minorías étnicas), como por la complejidad de las problemáticas, como es el caso de personas con problemas de adicciones, que requieren de una intervención interdisciplinar (Ortún-Rubio, Pinto-Prades y Puig-Junoy, 2001).

\section{Barreras estructurales}

Hay barreras estructurales del propio sistema de bienestar que a su vez interactúan con la cronificación de las situaciones de exclusión social grave. En el IV Estudio sobre la situación de las personas en situación de exclusión residencial grave en la CAPV 2018 (SIIS Centro de Documentación y Estudios, 2019), se observa que los factores de carácter estructural son barreras sociales que impiden la inclusión plena de las personas dentro del sistema de bienestar actual. Una de esas barreras es el acceso la vivienda. El acceso a vivienda de alquiler social es muy limitada y el alquiler libe es imposible ya que no se admite a personas que no presenten nómina o contrato laboral.

Otra barrera social es el acceso al sistema de salud en personas extranjeras (SIIS Centro de Documentación y Estudios, 2013). Como ejemplo se puede considerar que el $30 \%$ de las personas de 
origen extranjero, después de la entrada en vigor de la ley de servicios sociales de 2008 , no tiene tarjeta sanitaria, frente a un $3,7 \%$ de las personas no extrajeras.

\section{Incipiente consideración del impacto de género}

La situación de especial vulnerabilidad de las mujeres en situación de exclusión social grave responde al estigma intrínseco al mandato de género en nuestra sociedad. Las mujeres consumidoras sufren a veces el rechazo incluso entre los propios usuarios de drogas (Romo Avilés, 2005; Avilés y García, 2006; Aljama, Urbano y Santamaría, 2004). La historia de abuso de drogas en las mujeres está ligada a sucesos traumáticos que marcan su desarrollo personal y social posterior, por lo que las intervenciones deben proporcionar seguridad para restablecer un vínculo que pueda llegar a ser terapéutico (Hernán, Fernández y Ramos, 2004).

\section{La exclusión social grave afecta de manera especialmente desigual en función del sexo}

Las mujeres en exclusión social en Euskadi han vivido en mayor medida situaciones de abuso, y una mayoría de ellas han sido víctimas de agresiones sexuales. Tienen una mayor prevalencia de problemas relacionados con la salud mental, consumen drogas en menor medida y tienen una red de relaciones personales algo más amplia que los hombres (SIIS Centro de Documentación y Estudios, 2019). La dimensión traumática específica por razón de ser mujeres es también un eje común en las mujeres: un estudio de 2016 (Vázquez Cabrera et al., 2016) describe que, de las mujeres participantes, un 46,4\% se habían quedado embarazadas sin desearlo y un $58,7 \%$ habían padecido un aborto. Un $20,3 \%$ habían sido madres sin apoyo de su pareja.

Los hombres en mayor medida han dormido en la calle, han sido víctimas de delitos y amenazas, tienen un peor estado de salud física y una mayor prevalencia de enfermedades crónicas o graves, muestran mayor probabilidad de consumir drogas y mantienen menos contactos familiares. Estos datos se recogen en la Estrategia vasca de personas sin hogar 2018-2021, del Departamento de Empleo y Políticas Sociales del Gobierno Vasco (Gobierno Vasco, 2018). En el último recuento de personas sin hogar en Bizkaia, el porcentaje de mujeres viudas o solteras fue del $36,8 \%$, frente a un $17,6 \%$ de los hombres. En la categoría sin techo, las mujeres representan el $20 \%$, en exclusión residencial, o sin vivienda, las mujeres representan el $50 \%$. La dimensión relacional es el área más dañada en la historia de vida de las mujeres sin hogar. Precisamente por el rol social adjudicado a la mujer para el cuidado de otras personas en el ámbito privado, se sufren situaciones de abuso y violencia en la invisibilidad del hogar. Paradójicamente, esta victimización las puede llevar a una segunda victimización cuando se ven privadas del rol social adjudicado, sin hogar, sin familia, sin apoyos institucionales. Desde una perspectiva psicosocial, el término sinhogar no puede referirse a las personas que duermen en la calle; sino a todas aquellas personas que carecen de una vivienda digna, y que se ven abocadas a soportar situaciones de dependencia más allá de su seguridad y dignidad, con vulnerabilidad a la invisibilidad y aislamiento social.

Los servicios destinados a mujeres sin hogar se diferencian en dos tipos: servicios específicos para mujeres y generalistas. Existen elementos que dificultan la atención a las mujeres en servicios específicos: la escasez de centros y la orientación por perfiles (mujeres víctimas de violencia de género, y madres solteras), así como la falta de una atención integral.

\section{Escaso desarrollo de las prestaciones de tipo psicosocial}

Dentro del propio sistema de servicios sociales para la inclusión, también existen otras barreras estructurales (Zalakain, 2018). La prevalencia de trastornos y enfermedades mentales y trastornos adictivos en los servicios sociales para la exclusión social es muy alta. Esto les dejaría fuera del acceso a los servicios por razones meramente estructurales. Sin embargo los recursos sociosanitarios no contemplan la suficiente intensidad de la intervención para personas con esta compleja situación.

Los requisitos previstos para acceder a los servicios sociales, tales como tener conciencia del problema, motivación para el cambio, no precisar asistencia sanitaria especializada, o no rechazar el tratamiento si se trata de una enfermedad mental, son criterios que hay que cuidar especialmente en las personas con comorbilidad psiquiátrica y problemas de adicciones, que además están en situación de exclusión social grave. Para ello se requiere una intervención psicosocial o asistencia psicológica específica para prevenir la exclusión de estas personas del propio sistema de protección por exclusión social.

En personas en situación de exclusión social grave que presentan adicciones o alcoholismo, la falta de apoyo social, ligada a la precariedad de recursos, hace que el tratamiento de las adicciones se haga muy difícil, con el consiguiente agravamiento de la salud y la convivencia de estas personas en los recursos de acogida. La figura psicóloga profesional capaz de coordinar la intervención sociosanitaria sería esencial en la situación actual de los servicios sociales para la exclusión social.

En resumen, la perspectiva fragmentaria y departamentalizada, las barreras estructurales y las barreras inherentes al escaso desarrollo del propio 
sistema de servicios sociales son los principales retos que requieren de nuevos modelos e impulsos. Las prestaciones de carácter psicosocial pueden servir de gran ayuda a la mejora de la calidad de vida, autonomía e integración de las personas en los servicios sociales para la inclusión.

Los modelos que incidan en un acompañamiento social de calidad, multidisciplinar y basado en derechos y necesidades podrán contribuir a que el espíritu innovador de la ley de servicios sociales se plasme de hecho en la práctica de acompañamiento en la intervención social (Gobierno Vasco, 2008).

\section{El modelo de acompañamiento psicosocial a la integración social Integra/ Erantsi}

\subsection{Justificación}

El modelo Integra/Erantsi de acompañamiento a la integración social, está orientado a trabajar con personas con comorbilidad psiquiátrica y/o por drogodependencias, o a personas con dificultades de integración social por causa de discapacidad, dependencia, o aislamiento social (Vicioso y CastilloGonzález. 2019a) ${ }^{1}$.

El modelo de acompañamiento propuesto se concreta en un programa de baja exigencia, de carácter no directivo, que desde una perspectiva sociocomunitaria brinda atención y apoyo emocional a familiares, usuarios y usuarias en clave de acompañamientos individuales y grupales, promoviendo un vínculo terapéutico basado en la autonomía y la participación de la persona usuaria en la toma de decisiones acerca de su proceso de inserción social.

El énfasis recae en los procesos relacionales y comunicativos como componentes básicos del proceso de acompañamiento. Este acompañamiento es multinivel. A nivel individual, se basa en la relación de ayuda, un vínculo que se refuerza a través del compromiso y el refuerzo mutuo hacia la autonomía. En un segundo nivel, el acompañamiento se desarrolla en grupo, que es el que da sentido y soporte a todo el proceso.

En este sentido este modelo de acompañamiento psicosocial pretende adaptar la atención a personas con historias de vida complejas, y que presentan dificultades en el cumplimiento y mantenimiento en el sistema en caso de no recibir un apoyo extra.

Algunos de los criterios que pueden requerir de este tipo de acompañamiento psicosocial son los siguientes:

${ }^{1}$ La guía del modelo está disponible en 〈https://www.euskadi. eus/contenidos/informacion/publicaciones_ss/es_publica/adjuntos/GUIA_ERANTSI.pdf).
- No tener conciencia de su situación ni motivación para cambiarla.

- Precisar un acompañamiento psicológico para procesos psiquiátricos, cuidados paliativos, tratamiento crónico por adicciones, covid-19 0 VIH.

- Dificultades psicológicas para aceptar el tratamiento psiquiátrico o por adicciones, por VIH o por cáncer.

- Precisar un acompañamiento específico como mujer víctima de violencia de género o historial traumático.

- Precisar un acompañamiento específico como personas a cargo de menores o mayores dependientes.

\subsection{Principios básicos del modelo}

Este modelo de acompañamiento psicosocial Integra/ Erantsi, se dirige a las personas usuarias de servicios sociales para la exclusión social, especialmente aquellas personas en situación de exclusión social grave y con comorbilidad psiquiátrica y/o por drogodependencias.

Se considera que las intervenciones irán diseñadas constatando las necesidades específicas de las mujeres, con un enfoque de discriminación positiva, donde haya prestaciones específicas para mujeres tanto en el nivel individual como grupal, convivencial y sintónico con las sensibilidades específicas y centradas en la persona como mujer.

Tal y como se recoge en la Ley 4/2005 de Igualdad de Mujeres y Hombres de Euskadi, en su artículo 45, que hace referencia a la inclusión social (De Viergol, 2005):

Las administraciones públicas vascas adoptarán las medidas necesarias para contrarrestar la mayor incidencia de la pobreza y la exclusión social en las mujeres. Y ello, por una parte, a través de la integración de la perspectiva de género en los diferentes programas sectoriales dirigidos a garantizar a todas las personas el disfrute de los derechos sociales básicos y, por otra, mediante el diseño de programas específicos para colectivos de mujeres que sufren discriminación múltiple.

El acompañamiento psicosocial implica la coordinación y coparticipación de áreas de intervención social y sanitaria, desde el nivel inicial de autocuidado hasta el nivel más especializado (Vicioso y Castillo-González. 2019a). Además, puede actuar como puente con los servicios de atención especializada, para la derivación urgente en situaciones de crisis o la detección temprana de recaídas o brotes. Puede ampliar la cobertura de los servicios y llegar a un mayor número de personas que no tienen familia. 
El modelo se basa en la atención directa a la persona a través del vínculo individual y grupal, así como la atención hacia los vínculos con elementos comunitarios y sociales en el contexto actual de la persona. Centra su atención en la mejora de las competencias como estrategia educativa y terapéutica para compensar los déficits personales y sociales. Este modelo centrado en la persona hace especial énfasis en la mejora del bienestar psicosocial subjetivo y objetivo.

El acompañamiento psicosocial está dirigido a mejorar la calidad de vida y bienestar psicosocial de las personas que se encuentran en situación de exclusión social grave con comorbilidad psiquiátrica y/o por adicciones, favoreciendo su autonomía funcional e integración relacional.

El uso de drogas es un tipo de comportamiento que tiene un carácter multidimensional (desde la abstinencia hasta el abuso continuo, pasando por el consumo esporádico), el proceso individual de desarrollo de una adicción está multifactorialmente determinado. Tanto factores biológicos, como psicológicos y sociales, interactúan en la aparición, mantenimiento y agravamiento de la conducta adictiva (Iglesias y Tomás, 2011). Las personas en situación de exclusión social grave con historial de abuso de drogas presentan en su mayoría salud deteriorada, adicción crónica y frecuentes recaídas.

El acompañamiento a personas con problemas de adicciones desde los servicios sociales establecido en la CAPV se recoge en la Ley $1 / 2016$, de 7 de abril, de Atención Integral de Adicciones y Drogodependencias (BOPV, 2016). Son objetivos generales de la ley los siguientes:

a. Mejorar la salud y la calidad de vida de las personas con problemas por consumos de drogas y otras sustancias con capacidad adictiva, así como por las adicciones comportamentales, especialmente en el caso de quienes se encuentran en situación de vulnerabilidad o exclusión.

b. Facilitar la integración y la inclusión de las personas con adicciones tanto a drogas y otras sustancias con capacidad adictiva como por las adicciones comportamentales.

Se reconoce el derecho de las personas consumidoras a la igualdad de oportunidades de acceso a los servicios sociales. Cuando estas sean usuarias de servicios sociales, de servicios de salud o de servicios sociosanitarios, disfrutarán de los derechos reconocidos para las personas usuarias de tales servicios en las leyes sectoriales vigentes, tanto de servicios sociales como de salud y en sus disposiciones de desarrollo. Asimismo, deberán cumplir con las obligaciones establecidas para las personas usuarias en dichas normas.

El Sistema Vasco de Servicios Sociales, en su atención a las personas con adicción o con riesgo de padecerla, se aplicará de forma prioritaria: a. a la prevención de las causas que conducen a la exclusión social de las personas con adicción 0 riesgo de padecerla;

b. a la promoción de la autonomía y de la inclusión social de las personas que presentan adicción o riesgo de padecerla; $y$

c. a la atención a las personas con adicción o riesgo de padecerla, en el contexto del Catálogo de Prestaciones y Servicios Sociales, mediante la articulación, en el marco de un plan de atención personalizada, del conjunto de servicios o prestaciones económicas más idóneas.

En el modelo de acompañamiento psicosocial Integra/Erantsi, la adicción se comprende dentro de un sistema disfuncional, desde el plano motivacional, como un intento evasivo de automedicación en personas con tendencias adictivas como rasgo de personalidad. Pone énfasis también en los modelos de organización familiar y la relación con los servicios de atención profesionales (Stanton y Todd, 2018).

\subsection{La relación de ayuda en el modelo de acompañamiento psicosocial a la integración social}

El objetivo de este modelo Integra/Erantsi es facilitar el apoyo emocional a través del acompañamiento a lo largo del proceso de inclusión social. La metodología propuesta está basada en la experiencia de acompañamiento terapéutico individual y grupal llevada a cabo por la asociación T4 de lucha contra el sida de Bilbao, durante los últimos cinco años. La clave de la intervención está en manejar los procesos relacionales y comunicativos como componentes básicos del proceso de acompañamiento.

La intervención psicosocial está basada en la relación de ayuda, que tiene como objetivo mejorar el bienestar psicológico de la persona. Las tres características fundamentales sobre la relación de ayuda son su naturaleza colaborativa, el vínculo, y los objetivos consensuados. La condición previa es la colaboración; es decir, una propuesta de trabajo por parte de la persona. Es lo que se conoce como demanda explícita y manifiesta por parte de la persona que solicita la ayuda. Ha de ser una demanda o solicitud de ayuda consciente voluntaria y autónoma, y si se tratase de una persona no autónoma, como pudiera ser una persona menor de edad, o alguien con discapacidad, se consideraría como demandante al tutor de esa persona. La segunda característica es que se trata de un vínculo significativo, previsible, disponible en el tiempo y en el espacio, estable y personalizado. La relación de ayuda es primariamente sostenedora y proporciona seguridad a través de la disponibilidad emocional para validar el malestar de la otra persona y facilitarle a través de la relación los límites y el apoyo necesario para su desarrollo personal.

El vínculo debe mostrase en una actitud permanente de disponibilidad suficientemente buena, en el punto medio entre la protección y la delegación de responsabilidades, entre el afecto y la firmeza. 
Supone un compromiso emocional, basado en la comprensión y acompañamiento de la persona que demanda la ayuda. Esta característica se basa en los principios de la relación de ayuda, que son la congruencia, la aceptación, la incondicionalidad y la empatía (Rogers, 2012).

La tercera característica es que los objetivos a alcanzar tienen que ser consensuados, negociados y renegociados en una construcción. No se puede ir más allá de lo que la persona quiere cambiar o revisar en su proceso.

Los objetivos sirven de guía para evaluar el proceso de acompañamiento psicosocial.

El apoyo psicosocial será de mayor a menor intensidad en función de las necesidades y se podrá ir variando, deseablemente reduciendo la intensidad del acompañamiento requerido. Puede ser de varios tipos:

- Apoyo instrumental: información, orientación y mediación para el acceso a servicios de otros sistemas así como a redes sociofamiliares de apoyo.

- Apoyo emocional: reducción del daño, contención, soporte emocional, y orientación en situaciones de crisis.

- Apoyo relacional: escucha activa, adquisición de habilidades para mantener o establecer relaciones sociales, $y / 0$ personales, generando oportunidades para el establecimiento de relaciones.

\subsection{Objetivos del modelo de acompañamiento psicosocial a la integración social}

El proyecto pretende adaptar estrategias de acompañamiento psicosocial a personas con historias de vida complejas y situaciones de extrema gravedad dentro de la exclusión social. Se pretende llegar al objetivo último de mejorar la autonomía funcional y la integración relacional, propio de la inclusión social, a través de objetivos intermedios.

- Mejorar la calidad de vida. Se comprende la calidad de vida en su sentido más amplio; como recogen los autores Schalock y Verdugo (Schalock, 2014), las dimensiones de calidad de vida son: bienestar emocional, relaciones interpersonales, bienestar material, desarrollo personal, bienestar físico, autodeterminación, integración social, derechos, e integración comunitaria.

- Fomentar la autorrealización. Acompañar a personas que presentan necesidades psicosociales complejas asociadas a historial de adicciones o problemas de salud mental, supone ayudar a desarrollar la autorrealización (Ryff y Singer, 2008): autocuidados, autoconocimiento, bienestar emocional, relaciones positivas con los demás y crecimiento personal.

- Mejorar el bienestar social, tanto en ajuste social como en apoyo social percibido. El bienestar social es otra dimensión importante, ha sido definido como "la valoración que hacemos de las circunstancias y el funcionamiento individual dentro de la sociedad” (Keyes, 1998), y está compuesto por las siguientes dimensiones:

- Integración social o evaluación de la calidad de las relaciones que mantenemos con la sociedad y con la comunidad. La integración social implica sentirse parte de la comunidad, a través de lazos de apoyo y colaboración con las redes comunitarias. Podemos asociarlo con la dimensión de relaciones sociales positivas.

- Aceptación social. Se refiere al grado de disfrute que se experimenta a partir de saberse y sentirse perteneciente $a$ un determinado grupo o comunidad. Implica la aceptación de los aspectos positivos y negativos de la vida. En conjunto, ambos aspectos son indicadores de salud mental. La aceptación social indica que la persona tiene una actitud positiva hacia el resto de los seres humanos, aunque a veces la conducta sea compleja o incomprensible. Puede tener una relación con la autoaceptación.

- Contribución social. Se refiere al sentimiento de ser parte importante en la sociedad; de tener algo positivo que ofrecer al mundo y que las actividades personales son valoradas: "Yo tengo algo de valor que aportar al mundo" (Keyes, 1998), lo que facilita la motivación y los propósitos en la vida. Se percibe que la relación con el entorno social facilita tener metas y propósitos en la vida.

- Actualización social. Se refiere a la confianza en el potencial de crecimiento y desarrollo de una sociedad, así como de su capacidad para generar bienestar para quienes forman parte de ella. Se asocia a percibir que el entorno permite el crecimiento personal.

- Coherencia social: Se refiere a la capacidad de entender la organización y funcionamiento del mundo social. Puede equipararse a la dimensión de dominio del entorno.

\subsection{Metodología}

La metodología de acompañamiento psicosocial Integra/Erantsi se estructura en cuatro fases, según el desarrollo de la relación de ayuda (estas fases no son exclusivamente lineales o cronológicas; sirven para ordenar las actuaciones, pero se pueden ir retroalimentando a lo largo del proceso relacional).

- Fase Inicial: acogida o fase de creación de vínculo.

- Fase exploratoria: establecimiento de las bases de alianza y compromiso de cambio.

- Fase valorativa: narración resumida de la exploración y valoración de los elementos 0 factores individuales y contextuales, actuales e históricos, extraídos de profesional y en colaboración con la persona usuaria.

- Fase de intervención: elaboración del plan de intervención de manera colaborativa. 
El fin último que persigue el acompañamiento psicosocial es que los procesos individuales se realicen desde una perspectiva multidimensional e integradora, con el empleo de herramientas técnicas psicológicas.

\subsubsection{Fase inicial: acogida o fase de creación de vínculo}

La acogida se basa en la disponibilidad para la escucha (Vicioso y Castillo-González, 2019a). En estos primeros encuentros se pretende la clarificación inicial acerca de la función del acompañamiento psicosocial. En esta fase se establecen las bases de la relación de ayuda: confidencialidad, constancia, actitud contenedora, y establecimiento de límites claros para la persona y para su entorno. La persona usuaria se compromete a acudir a todas las citas con puntualidad y, en el caso en el que no fuera posible, avisar con anterioridad. Para ello se firma un contrato de acompañamiento por el que todas las partes se comprometen a salvaguardar los compromisos en términos de derechos y obligaciones. Los contenidos o áreas de intervención que pueden plasmarse en dicho contrato de acompañamiento psicosocial son:

- Autonomía: cada persona se responsabiliza de su proceso de desarrollo personal.

- Emociones: no se puede actuar de manera agresiva ni hostil durante el proceso de acompañamiento, ni hacia el otro ni hacia uno mismo.

- Propósitos: el proceso de acompañamiento implica propósitos individuales y relacionales.

- Planificación: el proceso de acompañamiento se observa a través del establecimiento de metas personales e intrínsecas, y conlleva que cada persona se ponga sus propios objetivos realistas y observables.

- Objetivos personales: integración de una imagen positiva de sí mismo. Fortalecimiento de la autoestima y autonomía. Incremento de la autorregulación afectiva y autoconocimiento.

- Relaciones: el proceso de acompañamiento psicosocial implica aumentar la red de relaciones sociales significativas o mejorar las relaciones con las personas que forman esa red.

- Objetivos relacionales: integración en la comunidad y mejora de la capacidad de empatía y asertividad.

\subsubsection{Fase valorativa: exploración y formulación del problema}

La exploración de los problemas y sus factores asociados pretende focalizar en las cuestiones prioritarias o problemas principales que preocupan a la personas y se asocian a su malestar emocional. Para ello se realiza una exploración de la situación biopsicosocial actual y pasada. La exploración permite recabar una información integral y general sobre los distintos ámbitos de autonomía e integración relacional:

- Salud mental general: historia psiquiátrica, interacción entre los síntomas en salud mental y uso de drogas, identificar periodos de estabilidad, periodos de cambio y periodos de crisis.

- Salud física general: breve historia médica (tratamientos, ingresos, bajas, diagnósticos).

- Situación familiar: historia médica y psiquiátrica familiar.

- Situación social: situación financiera, nivel educativo, historia laboral, ajuste social (red social de apoyo, arraigo).

- Evaluación del contexto: red de servicios intervinientes en la actualidad, relación con la comunidad, apoyo formal.

- Evaluación de las competencias: puntos fuertes, intereses y hobbies.

A partir de la información objetiva y las impresiones subjetivas recogidas, se construye la formulación del problema con la participación de la persona usuaria. Dicha formulación del problema integra en un todo coherente, información relativa a factores biológicos, psicológicos y sociales asociados al malestar. La formulación consiste en una clarificación de aspectos esenciales detectados y la relación existente entre ellos:

- Quién realiza la demanda.

- Cuál es la demanda.

- Características culturales y otras variables que inciden en la situación presentada.

- Problemas principales: detectar prioridades.

- Factores históricos, contextuales y estructurales.

- Quiénes son los destinatarios y las destinatarias de esta formulación: persona, familia, equipo.

- Clarificación y consenso de las hipótesis e inferencias teóricas a partir de la información registrada: problemas manifiestos y factores asociados (modelo multiteórico de caso -Eells, 2011-).

La formulación en las personas con adicciones se basa en el modelo biopsicosocial y sistémico. De acuerdo con este, los componentes esenciales para explicar la iniciación, mantenimiento o abandono de la conducta adictiva son la vulnerabilidad individual, el contexto y las consecuencias (Secades-Villa et al., 2007). La motivación para el cambio debe ser necesariamente abordada desde una perspectiva psicológica.

\subsubsection{Fase de intervención: elaboración del plan de intervención de manera colaborativa}

En esta fase se trata de elaborar un plan de acción de acompañamiento psicosocial en base a: 
- La integración relacional y el nivel de autonomía funcional.

- La existencia de comorbilidad por uso de drogas/ comorbilidad psiquiátrica/exclusión social.

- La formulación previamente establecida de la relación entre todos los aspectos individuales y contextuales considerados.

El diseño del plan de intervención se basa en las siguientes premisas:

- Apoyo y supervisión de un equipo estable y una organización explícitamente formulada: número de sesiones por caso, periodicidad, y duración de estas.

- Participación de las personas usuarias en el desarrollo de un modelo de acompañamiento psicosocial colaborativo que permita que las personas se sientan comprendidas y que les permita experimentar su participación activa en el proceso.

- Consideración de la experiencia vivida por las personas y su historia biográfica, validación de la experiencia subjetiva de la persona, a la vez que se abren nuevas perspectivas y modos de experimentar y percibir dichas experiencias.

- Contención clara y compromiso de no hacerse daño. El riesgo de daños debe ser considerado por las personas acompañantes y por la organización para prevenirlos y contener las crisis cuando se produzcan.

\subsection{Estrategias de acompañamiento psicosocial en personas en situación de exclusión social grave}

\subsubsection{Apoyo psicosocial emocional}

Las competencias básicas para desarrollar el clima de apoyo emocional (Cuadro 2) permiten mantener las condiciones básicas de confidencialidad, seguridad, relación de colaboración, compromiso, flexibilidad, empatía, motivación para el cambio, y compromiso de no hacerse daño. Esas competencias se pueden resumir en: claridad y transparencia, validación, escucha activa, indagación, confrontación y contención (Cuadro 1).

Para potenciar la autenticidad, una de las competencias es la claridad y transparencia. Es necesaria una estructura clara, explícitamente formulada para que la persona sepa lo que le puede esperar, quién forma parte del equipo, el número de sesiones que se pueden llevar a cabo, qué se puede predecir, qué no se puede predecir, qué compromisos se esperan por su parte, qué intervención puede haber con respecto a las personas que puedan estar en su entorno.

También es importante para manifestar la autenticidad, no confundir con la identificación o implicación personal. La neutralidad es tomar a la persona como válida y considerar el contexto social y cultural de la persona. Conocer el contexto social ayuda a comprender mejor sus conflictos y proporciona mayor neutralidad e imparcialidad.

Respecto a la condición de aceptación y consideración positiva incondicional la principal competencia a desarrollar es la validación. Es necesario validar continuamente de forma verbal y no verbal la experiencia de la persona. Es importante asumir como válidas dentro de la relación de ayuda todas las experiencias de forma explícita. Si se tratase de experiencias traumáticas también es necesario validar la importancia que tienen. Es necesario darle visibilidad a la experiencia vivida por la persona ya sea verbal o no verbalmente y así manifestar esa consideración positiva incondicional.

Las competencias para desarrollar la empatía principalmente son: una escucha activa, en el aquí y ahora, prestando una atención plena y comprometida a la persona y comunicando esa disposición, tanto en el lenguaje verbal como no verbal. La comprensión empática se manifiesta a través de las habilidades de comunicación con preguntas abiertas, indagación sobre patrones repetitivos de conducta, motivaciones, etc. Por último la competencia que se corresponde con la necesidad de establecer los límites es la capacidad de contención. La contención consiste en velar por el cumplimiento del contrato y el encuadre, constituyéndose en un espacio seguro donde la persona puede desplegar su dinámica de funcionamiento. Deben ser evaluadas y quedar explicitadas aquellas situaciones que puedan constituir una amenaza (por ejemplo, conductas autodestructivas y suicidas, consumo de sustancias, o amenazas). También han de darse algunas condiciones específicas, como por ejemplo establecer las reglas que van a regir: el compromiso de asistencia, el respeto de los tiempos, son todos elementos básicos que estructuran el acompañamiento emocional y permiten su progreso. La confrontación, por su parte, consiste en contrastar contradicciones o elementos del discurso que no son coherentes o que suponen una resistencia o falta de compromiso.

\begin{tabular}{|l|l|}
\hline Cuadro 1. Competencias en la relación de ayuda \\
\hline Condiciones & Competencias \\
\hline Autenticidad & $\begin{array}{l}\text { Claridad } \\
\text { Neutralidad }\end{array}$ \\
\hline $\begin{array}{l}\text { Aceptación, consideración } \\
\text { positiva incondicional }\end{array}$ & Validación \\
\hline Empatía & $\begin{array}{l}\text { Escucha activa } \\
\text { Clarificación } \\
\text { Señalamiento }\end{array}$ \\
\hline Establecimiento de límites & $\begin{array}{l}\text { Capacidad de contener } \\
\text { Confrontación }\end{array}$ \\
\hline
\end{tabular}




\subsubsection{El apoyo psicosocial grupal}

El grupo de soporte emocional se basa en la interacción a partir de la situación similar de partida de sus miembros. Las normas de funcionamiento crean el contexto de soporte emocional, y también la figura facilitadora, que se encarga de velar por el cumplimiento de las normas de funcionamiento, entrevistar a los nuevos miembros, coordinar las reuniones e identificar las dinámicas grupales puede reforzar los objetivos de la intervención gracias al apoyo entre iguales en grupos cerrados de tamaño pequeño (Sánchez Vidal, 1995). Se trata de crear un espacio de seguridad que permita la participación, así como una estructura clara que favorezca la orientación y la atención. Los principales compromisos son la asistencia y confidencialidad. La relación entre iguales, la expresión emocional en un ambiente seguro donde el compromiso y la filosofía de reciprocidad de la ayuda ofrecen un espacio para el encuentro y el apoyo social emocional.

En el grupo, el apoyo emerge de las personas participantes. La figura profesional vela por la integridad individual y protege a los miembros más vulnerables. Incentiva la dinámica grupal y la participación. Crea una atmósfera agradable tanto de trabajo como de relación entre sus miembros, favoreciendo la continuidad del grupo. Deja que el grupo se desarrolle según las necesidades de sus miembros. Es imprescindible que el facilitador o facilitadora sea una persona cualificada y además suele ser de gran ayuda la supervisión de otro profesional.

El elemento más importante es la confidencialidad, junto con los siguientes.

- La protección de los derechos individuales en el grupo.

- Conformidad con las normas definidas por el grupo como aceptables y adecuadas.

- No uso de drogas durante el grupo.

- No uso de violencia.

- Asistencia regular y justificación de la no asistencia.

- Todo lo del grupo corresponde al grupo.

- Todos los miembros del grupo deben aceptar y consensuar las normas establecidas.

El encuadre grupal prevé una periodicidad semanal o quincenal, manteniendo la hora y el día y el lugar estables. La estructuración de cada sesión puede variar en función del grupo, pero es importante que se contemple la cohesión grupal, fomentando la asistencia y la participación. Para ello se realizan o juegos de interacción, y al cierre, para que todas

las personas puedan expresarse al menos antes de finalizar cada sesión.

\subsubsection{Apoyo psicosocial a la salud}

En personas en situación de exclusión social grave y comorbilidad psiquiátrica y/o por adicciones, el apoyo psicosocial se plantea como una estrategia básica para mejorar su calidad de vida. El acompañamiento psicosocial implica un acompañamiento directo a la persona desde una perspectiva de reducción de daños, que incluye:

- Acompañamiento y supervisión de los tratamientos de mantenimiento con agonistas opiáceos. Hay evidencia que demuestra que, si bien la metadona funciona muy bien para la mayoría de pacientes, algunos de ellos no responden a este tratamiento, abandonándolo 0 continuando con el consumo de otros opioides en la calle (Iglesias et al., 2008). El apoyo psicosocial a las personas en programas de mantenimiento con agonistas opiáceos permite la adecuación progresiva de alternativas en caso de ser demandadas y la optimización de su eficacia.

- Apoyo a la utilización, en su caso, de salas de consumo supervisado. Las salas de consumo supervisado son recursos de atención sanitaria profesionalmente supervisadas, donde las personas usuarias de drogas pueden consumir en condiciones seguras e higiénicas. Se consideran un servicio altamente especializado y dentro de los servicios existentes para personas consumidoras. Los estudios para evaluar la eficacia de las salas de consumo desprenden resultados positivos respecto al alcance de la población diana y de la provisión de un entorno higiénico y saludable para el consumo de drogas (Schatz y Nougier, 2012).

- Acompañamiento en procesos de desintoxicación u hospitalización por causa de la adicción.

- Apoyo en el acceso y mantenimiento en programas libres de drogas.

- Apoyo a la adherencia, no solamente considerada como adherencia farmacológica, sino que incluye además los seguimientos médicos y psiquiátricos, citas médicas, controles y tratamientos médicos específicos. Se trata de facilitar estrategias conductuales de adecuación al tratamiento (información, planificación, monitoreo). Promover actitudes positivas hacia el tratamiento, emociones positivas, y aceptación de la condición de vivir con VIH. La mayoría de los estudios señalan que el tratamiento psicosocial mejora el pronóstico de los pacientes al mejorar la retención en los programas terapéuticos, aumentar los periodos de abstinencia, disminuir el consumo global y mejorar la comorbilidad (Martín, Hernández y Carrobles, 2005).

\subsection{Evaluación de la intervención psicosocial según el modelo Integra/Erantsi}

En los últimos años, la Asociación T4 de lucha contra el sida aplica el modelo de acompañamiento psicosocial para personas con VIH en situación de exclusión 
social y con patologías múltiples. Los programas de acompañamiento psicosocial promueven un mayor acceso de las mujeres a la intervención, propiciando medidas de intervención específicas para mujeres para mejorar la relación de ayuda.

El enfoque que se emplea para el desarrollo de la intervención es biopsicosocial, comunitario, humanista, personalizado, continuado e integral. Aunque el objetivo último es mejorar la autonomía y la calidad de vida, es necesario garantizar la estructura vital para que estas personas puedan mejorar la situación social y de salud. En el caso del VIH, si por causas directa o indirectamente asociadas a la enfermedad se generan situaciones de exclusión grave, generalmente por carecer de apoyo familiar o carecer de vivienda, se requiere atención residencial con el fin de promover la integración social, la calidad de vida y la autonomía en un ambiente de protección de la salud. Otro factor de riesgo para mantener la adherencia y la calidad de vida son las alteraciones emocionales, la falta de motivación, o la evitación de afrontar los cuidados de la salud como mecanismo de negación.

En el año 2019, se lleva a cabo la evaluación del programa de acompañamiento psicosocial en treinta y tres personas usuarias del centro diurno y centro residencial para personas en situación de exclusión social de la Asociación T4. De los 33 participantes, 13 son mujeres y 23 son hombres (Vicioso y Castillo-González, 2019b). Todas las personas participantes están en situación de exclusión social. Son perceptoras de pensiones mínimas o renta de garantía de ingresos. Su salud es precaria, por problemas asociados al VIH, hepatitis y/o coinfección, adicción a sustancias tóxicas y/o abuso de estas, y enfermedad mental. Son en su mayoría mayores de cincuenta años. La mayoría de ellas han contraído el VIH; están en tratamiento psiquiátrico (50\%) o tratamiento de metadona (50\%).
Las variables medidas para evaluar la autonomía funcional y la integración relacional en las personas que participan en dicho estudio fueron las siguientes:

- Ajuste psicoafectivo. Afrontamiento conductual y emocional (Font y Cardoso, 2009).

- Apoyo social. Esta variable mide el nivel de apoyo social percibido. Se mide a través del Cuestionario MOS de Apoyo Social (Requena, Salamero y Gil, 2007). Adaptado de MOS-SS Medical Outcomes Study-Social Support Survey (Sherbourne y Stewart, 1991). Tiene una subescala de apoyo social estructural, que mide la red natural e informa de apoyo, y una subescala de apoyo social funcional, que mide apoyo social instrumental, relacional, emocional y afectivo.

- Ajuste social. Esta variable recoge el grado de satisfacción subjetiva de la persona en sus relaciones sociales, trabajo, ocio, participación comunitaria, motivaciones, hobbies e interés por el entorno. Se mide a través de la Escala de Satisfacción con la Adaptación Social (SAAS) (Bobes et al., 1999).

- Calidad de vida. Cuestionario MOS VIH (Remor, 2002).

- Adherencia farmacológica subjetiva. Cuestionario de Adherencia al Tratamiento Antirretroviral (CEATVIH) (Remor, 2006).

Los resultados de la evaluación se muestran en el Gráfico 1. La puntuación se considera baja si la media es inferior a $\mathrm{PC}=60$; media si el $\mathrm{PC}=60-80$; y alta si el PC medio es superior a $80^{2}$. La adherencia media al tratamiento médico y psiquiátrico y el ajuste social son óptimas (PC = 76) (Remor, 2006). La calidad de vida y el apoyo social percibido son también adecuadas $(P C=66)$ (Remor, 2002).

\section{Gráfico 1. Variables de integración relacional y autonomía funcional}

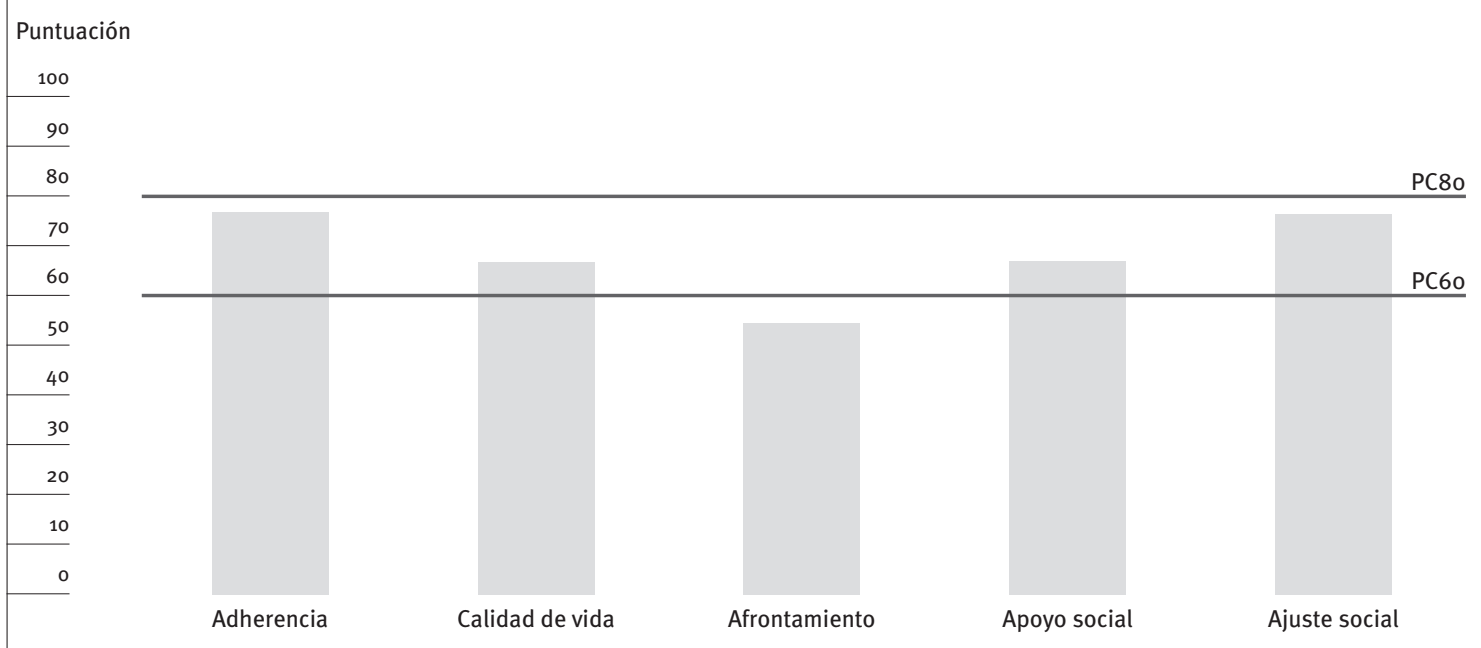

Fuente: Elaboración propia. 
El ajuste psicoafectivo, medido por las variables de afrontamiento emocional y conductual, es bajo: $P C=53$ y $P C=54$, respectivamente (Font Guiteras, Reventós Osuna y Falo Zamora, 2020).

La dimensión de apoyo social total incluye apoyo afectivo, instrumental, emocional e interacción social. Se obtiene una media de $X=59,37$, que es equivalente al $P C=68$, considerado adecuado para valores de media superiores a 57 (Requena, Salamero y Gil, 2007).

Se obtiene un nivel de ajuste social alto $(X=38,63)$, equivalente a un percentil de $P C=80$, siendo las puntuaciones más altas en las relaciones sociales y las actividades ocupacionales (Bosc, Dubini y Polin, 1997).

Si analizamos el perfil de las mujeres en este estudio piloto, se observa que todas ellas son usuarias de la red de servicios sociales de larga duración. A lo largo de su proceso vital han participado en diferentes servicios y programas de intervención social. En las mujeres la adherencia y calidad de vida es similar a la obtenida en hombres; sin embargo, obtienen ligeramente mejores resultados en ajuste social y apoyo social. El nivel de apoyo social informal o natural, que mide el tamaño de la red social presenta una media de $\mathrm{PD}^{3}=4,13$ personas significativas cercanas. Se considera apoyo social bajo cuando la puntuación media es inferior a PD $=5$ (Requena, Salamero y Gil, 2007).

Como limitación del estudio evaluativo realizado, cabe señalar que no se ha analizado el impacto de esta intervención específicamente con mujeres desde una perspectiva longitudinal. Los servicios destinados a mujeres sin hogar se diferencian en dos tipos: los servicios específicos para mujeres y los generalistas. Existen elementos que dificultan la atención a las mujeres en servicios específicos: la escasez de centros, la orientación por perfiles (mujeres víctimas de violencia de género y madres solteras), y la falta de una atención integral. En general, es necesario realizar estudios longitudinales para poder establecer relaciones causales o correlacionales entre los factores de apoyo social, ajuste social, calidad de vida y afrontamiento con las intervenciones de tipo psicosocial en personas con múltiples patologías asociadas a procesos de exclusión social.

\section{Conclusiones}

El modelo de acompañamiento psicosocial Integra/ Erantsi está destinado a personas que tienen múltiples problemáticas de salud y sociales asociadas a un historial de adicciones o a un historial psiquiátrico, que acompaña a su situación de exclusión social, y que hace más difícil su progreso hacia una mayor integración.

Estas problemáticas de salud están en continua interacción con el proceso de acompañamiento. Se produce un efecto sindémico de los factores de riesgo tales como la desestructuración personal, familiar o social. La interacción de estos factores produce una escalada y espiral hacia un agravamiento y cronificación de los problemas a nivel individual (salud mental), familiar (conflictos, rupturas), o social (exclusión, aislamiento). Es necesario establecer herramientas que permitan profundizar en las áreas más sensibles en el acompañamiento social a personas con patologías múltiples.

Las necesidades de intervención en el área de los servicios sociales son reconocidas como un derecho subjetivo de ciudadanía y se valoran en función de una visión integral de la persona. Lo que interesa a los servicios sociales es la interacción entre las capacidades (o limitaciones) funcionales para la vida diaria y los soportes relacionales (o su ausencia) por parte de los entornos familiares y comunitarios en los que tiene lugar esa vida diaria.

Aquellas personas que no acceden al sistema de bienestar pueden sufrir procesos de exclusión social y deterioro de su salud mental cada vez más complejos.

Se puede realizar una intervención en general $y$, específicamente, que contemple de manera relacionada los aspectos de exclusión social, salud mental y adicciones. 
ALJAMA, A.; URBANO, A. y SANTAMARÍA, E. (2004): La mujer drogodependiente: especificidad de género $y$ factores asociados, Universidad de Deusto.

AVILÉS, N.R. y GARCÍA, E.G. (2006): “Género y uso de drogas. De la ilegalidad a la legalidad para enfrentar el malestar". Trastornos adictivos, vol. 8, no. 4, pp. 243-250.

BOBES, J.; GONZÁLEZ, M.P.; BASCARAN, M.T.; COROMINAS, A.; ADAN, A.; SÁNCHEZ, J. y SUCH, P. (1999): "Validation of the Spanish version of the social adaptation scale in depressive patients", Actas españolas de psiquiatría, vol. 27, no. 2, pp. 71-80.

BURGOS, A.H.; ÁLVAREZ, F.M.; BLÁZQUEZ, A.M. y MAIZA, J.O. (2019): "La actual ley vasca de servicios sociales. Universalización de servicios y consolidación de la atención socioeducativa y de los educadores y educadoras sociales en las respuestas a la ciudadanía", RES: Revista de Educación Social, no. 29, pp. 43-69.

CASADO, D. (2010): Leyes de Servicios Sociales del siglo XXI, Cáritas Española.

SIIS CENTRO DE DOCUMENTACIÓN Y ESTUDIOS (2013): /I Estudio sobre la situación de las personas en situación de exclusión residencial grave en la CAPV.

DE VIERGOL LANZAGORTA, A.M. (2005): “Ley 4/2005, para la Igualdad de Mujeres y Hombres de la Comunidad Autónoma del País Vasco y propuesta de 'lege ferend' sobre el IV PAPME”, Revista técnico laboral, vol. 27, no. 105, pp. 433-464.

DIPUTACIÓN FORAL DE BIZKAIA (2019): Decreto Foral de la Diputación Foral de Bizkaia 59/2019, de 21 de mayo, por el que se regula el procedimiento de intervención especializado en materia de inclusión social, Cuadernos del Departamento de Empleo, Inclusión Social e Igualdad, Disponible en: 〈http://biblioteca.ararteko.eus/ Record/22410>.

EELLS, T.D. (2011): Handbook of psychotherapy case formulation, Guilford Press.

FANTOVA AZCOAGA, F. (2007): “Repensando la intervención social”, Documentación social, vol. 147, pp. 183-198.

- (2017): Servicios sociales e inclusión social: análisis y perspectivas en el País Vasco, Zerbitzuan, no. 64, pp. 9-31, 〈https://doi.org/10.5569/11347147.64.01>.

- (2018): “Construyendo la intervención social”, Papeles del psicólogo, vol. 39, no. 2, pp. 81-88.

FERNÁNDEZ, S. (2017): “Análisis de la realidad de las mujeres en situación o riesgo de exclusión social en la Comunidad Autónoma de Euskadi”, Zerbitzuan, pp. 141-152, 〈https://doi. org/10.5569/1134-7147.64.09>.

GOBIERNO VASCO (2008): Ley 12/2008, de 5 de diciembre, de Servicios Sociales.

- (2013): Decreto 385/2013, de 16 de julio, disponible en: <http://www.jusap.ejgv. euskadi.eus/r47-bopvapps/es/bopv2/ datos/2013/08/1303530a.pdf >.

- (2016): Ley 1/2016 de Atención Integral de Adicciones y Drogodependencias, 2016.

- (2017): Decreto 152/2017, de 9 de mayo.

- (2018): Estrategia Vasca para Personas sin Hogar, disponible en: 〈http://www.euskadi.eus/ contenidos/documentacion/doc_sosa acuerdo_sinhogar/es_def/adjuntos/ ESTRATEGIA VASCA PARA PERSONAS SIN HOGAR 2018-2021 castellano.pdf〉. 
HERNÁN, M.; FERNÁNDEZ, A. y RAMOS, M. (2004): “La salud de los jóvenes”, Gaceta Sanitaria, vol. 18, pp. 47-55.

IGLESIAS, E.B. y TOMÁS, M.T.C. (2011): Manual de adicciones para psicólogos especialistas en psicología clínica en formación, Socidrogalcohol.

IGLESIAS, E.B.; TOMÁS, M.C.; PÉREZ, E.J.P.; HERMIDA, J.R.F.; FERNÁNDEZ, L.C.; GONZÁLEZ, M.P.B.; VILLA, R.S. y GRADOLÍ, V.T. (2008): Guía clínica de intervención psicológica en adicciones, Socidrogalcohol.

KEYES, C.L.M. (1998): "Social Well-Being”, Social Psychology Quarterly, vol. 61, no. 2, pp. 121140, 〈https://doi.org/10.2307/2787065〉, disponible en: 〈http://www.jstor.org/ stable/2787065>.

KORTABARRIA, B.L.; ARRÁZOLA, F.J.L.; ZALAKAIN, J. y ÁLVAREZ, N.Z. (2018): "Elkar-EKIN:(re) construyendo en común la red de ser vicios sociales para la inclusión en Gipuzkoa", Zerbitzuan, no. 67, pp. 51-65, 〈https://doi. org/10.5569/1134-7147.67.04>.

LÓPEZ-AROSTEGI, R. (1970): “L'emancipació com a horitzó de la intervenció social”, Educació social. Revista d'intervenció socioeducativa, vol. o, no. 56, disponible en: 〈https://www. raco.cat/index.php/EducacioSocial/article/ view/275578>.

MARTÍN, L.L.; HERNÁNDEZ, S.O. y CARROBLES, J.A. (2005): "Variables psicosociales en la adherencia al tratamiento antirretroviral en pacientes adscritos a un programa de mantenimiento con metadona", Psicothema, vol. 17, no. 4, pp. 575-581.

ORTÚN-RUBIO, V.; PINTO-PRADES, J.L. y PUIG-JUNOY, J. (2001): "La economía de la salud y su aplicación a la evaluación”, Atención Primaria, vol. 27 , no. 1 , pp. 62-64.

REMOR, E. (2002): “Apoyo social y calidad de vida en la infección por el VIH”, Atención primaria, vol. 30 , no. 3, pp. 143-148.

- (2006): “Cuestionario para la evaluación de la adhesión al tratamiento antirretroviral (CEAT-VIH): estudio internacional", Rev Humanitas, vol. 3, pp. 80-81.

REQUENA, G.C.; SALAMERO, M. y GIL, F. (2007): “Validación del cuestionario MOS-SSS de apoyo social en pacientes con cáncer", Medicina Clínica, vol. 128 , no. 18 , pp. 687-691.

ROGERS, C.R. (2012): “La relación interpersonal: el núcleo de la orientación", Terapeutas y Psicólogos en CDMX: Irradia Terapia.

ROMO AVILÉS, M.N. (2005): Género y uso de drogas: la invisibilidad de las mujeres.

RYFF, C.D. y SINGER, B.H. (2008): "Know thyself and become what you are: A eudaimonic approach to psychological well-being", Journal of happiness studies, vol. 9, no. 1, pp. 13-39.
SÁNCHEZ VIDAL, A. (1995): "Potencial y límites de la intervención psicosocial”, Revista sobre Igualdad y Calidad de Vida, vol. 4, no. 10, pp. 5-15.

SCHALOCK, R.L. (2014): "Quality of life as a change agent”, International Public Health Journal, vol. 6, no. 2, pp. 105.

SCHATZ, E. y NOUGIER, M. (2012): Salas de consumo de drogas: evidencias y prácticas, IPDC.

SECADES-VILLA, R.; GARCÍA-RODRÍGUEZ, O.; FERNÁNDEZHERMIDA, J.R. y CARBALLO, J.L. (2007): "Fundamentos psicológicos del tratamiento de las drogodependencias", Papeles del psicólogo, vol. 28, no. 1, pp. 29-40.

SECO REVILLA, O. (2018): “Marco conceptual y caracterización del servicio de día para la inclusión social en Bizkaia”, Zerbitzuan, no. 67, pp. 67-78, 〈https://doi.org/10.5569/1134 7147.67.05>.

SHERBOURNE, C.D. y STEWART, A.L. (1991): "The MOS social support survey", Social science \& medicine, vol. 32, no. 6, pp. 705-714.

SIIS CENTRO DE DOCUMENTACIÓN Y ESTUDIOS(2019): IV Estudio sobre la situación de las personas en situación de exclusión residencial grave en la CAPV 2018, Vitoria-Gasteiz, Departamento de Empleo y Políticas Sociales. Disponible en: 〈http://www.siis.net/documentos/ informes/545454.pdf $>$.

STANTON, M.D. y TODD, T.C. (2018a): Terapia familiar del abuso y adicción a las drogas, Editorial Gedisa.

- (2018b): Terapia familiar del abuso y adicción a las drogas, Editorial Gedisa.

VÁZQUEZ CABRERA, J.J.; RODRÍGUEZ MORENO, S.I.; ROCA MORALES, P. y PANADERO HERRERO, S. (2016): "Sucesos vitales estresantes en mujeres en situación sin hogar”, Mujeres e investigación. Aportaciones interdisciplinares: VI Congreso Universitario Internacional Investigación y Género, pp. 611-624.

VICIOSO, C. y CASTILLO-GONZÁLEZ., D. (2019롤: Guía De Acompañamiento INTEGRA/ERANTSI para Personas en Procesos de Integración/ Gizarteratze Prozesuetan Diren Pertsonei Lagun Egiteko INTEGRA/ERANTSI Gida, Vitoria-Gasteiz, Departamento de Empleo y Políticas Sociales. Disponible en: 〈https://www.euskadi.eus/ contenidos/informacion/publicaciones_ss/ es_publica/adjuntos/GUIA_ERANTSI.pdf〉.

VICIOSO, C. y CASTILLO-GONZÁLEZ, D. (2019): Guía «INTEGRA-ERANTSI» de acompañamiento terapéutico para personas con vih. XIX Congreso Nacional sobre el Sida e ITS, Alicante.

ZALAKAIN, J. (2018): Pasado, presente y futuro de los Servicios Sociales para la Inclusión, Nuevos horizontes, Gizartegune. 